\title{
Diversité du peuplement ligneux d'une forêt dense en zone sub-humide : Cas de la forêt de Sakété dans le sud-Bénin en Afrique de l'Ouest
}

\author{
Jacques Boco ADJAKPA ${ }^{1 *}$, Hervé A. DASSOUNDO ${ }^{1}$, Hounnankpon YEDOMONHAN ${ }^{2}$, \\ Peter D. M. WEESIE ${ }^{3}$ et Elie L. AKPO ${ }^{4}$ \\ ${ }^{I}$ Département Génie de l'Environnement, EPAC/UAC, 01 BP. 2009 Cotonou (Bénin). \\ ${ }^{2}$ Département de Biologie végétale, FAST/UAC, 01 BP 4521 Cotonou (Bénin). \\ ${ }^{3}$ Science and Society Group, Faculty of Mathematics and Natural Sciences - University of Groningen. \\ ${ }^{4}$ Laboratoire d'Ecologie, FAST/UCAD, BP 5005 Dakar (Sénégal). \\ *Auteur correspondant, E-mail : adjakpaj@yahoo.fr ; Tél. (00229)95151464
}

\section{RESUME}

Cette étude réalisée en zone sub-humide présente les caractéristiques du peuplement ligneux de la forêt classée de Sakété située au sud-Bénin. La méthode de collecte des données sur la végétation a combiné la méthode d'inventaire floristique et celle dite de relevés itinérants. Le cortège floristique du peuplement ligneux actuel de la forêt classée de Sakété est constitué de 123 espèces réparties en 95 genres et 41 familles. Les Euphorbiaceae, les Leguminosae (16 espèces chacune) et les Rubiaceae (13 espèces) sont les mieux représentées. Trois types de formations végétales ont été définis : une forêt dense humide semi-décidue à Trilepiseum madagascariensis et Dichapetalum crassifolium, une forêt inondable à Anthonotha crassifolia et Symphonia globulifera et une forêt marécageuse à Anthostema aubryanum et Alchornea cordifolia. La richesse spécifique varie de 10 à 22 espèces ; les indices de diversité de Shannon et l'équitabilité de Pielou varient respectivement de 2,4 à 3,8 bits et de 0,5 à 0,8 . La densité du peuplement ( 80 à 200 individus/ha) est relativement faible et témoigne d'une exploitation anarchique. La surface terrière du peuplement a varié de 12,9 à $20,2 \mathrm{~m}$ /ha. La végétation de cet écosystème forestier, encore riche en espèces, présente cependant des signes de dégradation.

(C) 2011 International Formulae Group. All rights reserved.

Mots clés : Inventaire floristique, forêt classée, végétation, gestion durable.

\section{INTRODUCTION}

Les forêts ont de tout temps fourni aux Hommes, aux animaux et aux écosystèmes des biens et services parmi lesquels la protection et l'amélioration de la fertilité (hydrique et minérale) des sols (Akpo, 1993 ; 1998) pour la nutrition des plantes, la réduction de la pauvreté dans le monde rural, et la sécurité alimentaire (FAO, 2003) par divers produits forestiers, ligneux et non ligneux. Avec la forte poussée démographique, l'accroissement de la recherche d'espaces habitables et de terres cultivables par les Hommes, et/ou pâturables, et les besoins de plus en plus importants en énergie domestique, les stratégies d'exploitation des arbres, abondamment développées, ont réduit fortement les espaces boisés. 
Dans la zone guinéenne du Bénin, subsistent encore quelques îlots de forêts denses (Akoègninou, 2004) appartenant soit au domaine de l'Etat (les forêts classées), soit au domaine rituel (les forêts sacrées). Dans les forêts du domaine public, les ressources sont en proie à de fortes pressions anthropiques. Des exploitations illégales d'arbres pour produire du bois de feu, de charbon de bois ou $\mathrm{du}$ bois de services ont en effet réduit considérablement les effectifs de certaines espèces utiles. Malheureusement, peu de travaux de recherche ont porté sur ces milieux.

C'est dans ce contexte que cette étude sur les caractéristiques du peuplement ligneux de la forêt classée de Sakété a été menée dans le but de décrire l'état actuel de la forêt et de fournir des éléments de décision pour une gestion durable de cette forêt. Nous avons ainsi inventorié les espèces ligneuses, défini la structure des populations, identifié et décrit les différents types de communautés végétales.

\section{MATERIEL ET METHODES} Zone d'étude

L'étude a été menée dans la forêt classée de Sakété d'une superficie de 60 ha et située entre $6^{\circ} 40^{\prime}$ et $6^{\circ} 55^{\prime}$ de latitude Nord et $2^{\circ} 35^{\prime}$ et $2^{\circ} 45^{\prime}$ de longitude Est, à $1 \mathrm{~km}$ au Sud de la ville de Sakété (Figure 1). La forêt de Sakété fait partie du domaine forestier de l'Etat, qui a bénéficié du statut de forêt classée dans la région méridionale du Bénin depuis 1942. Le climat est de type subéquatorial marqué par deux saisons pluvieuses et deux saisons sèches (Akoègninou et al., 2006). La première saison de pluies s'étend d'avril à juillet avec un maximum de 183,9 mm en juin et la seconde de septembre à octobre avec un maximum de 141,3 $\mathrm{mm}$ en octobre. La première saison sèche s'étend sur le mois d'août et la seconde, de novembre à mars. La pluviosité moyenne annuelle est de 1112,7 $\mathrm{mm}$. La température est élevée tout au long de l'année. La moyenne annuelle est de 27,9 ${ }^{\circ} \mathrm{C}$. Les moyennes mensuelles varient de 25,6 ${ }^{\circ} \mathrm{C}$ à $30{ }^{\circ} \mathrm{C}$. Les sols sont de différents types et sont fonction du niveau topographique. Il s'agit : des sols ferralitiques (argileux, peu lessivés) sur les sommets des plateaux, de grès sur les pentes et des sols hydromorphes (sols sableux ou argileux à hydromorphie temporaire ou permanente) dans les endroits les plus bas (fond des dépressions).

\section{Méthode}

L'échantillonnage a utilisé des transects levés de la dépression au sommet de plateau ; trois niveaux topographiques ainsi définis (la dépression (bas-versant), la pente (mi-versant) et le sommet) ont été retenus pour servir de sites d'observation. Un placeau de $25 \mathrm{~m}$ x 20 $\mathrm{m}$ a été installé par niveau topographique le long de chaque transect.

La collecte des données sur la végétation a combiné la méthode d'inventaire floristique, la méthode de relevé de végétation et celle dite de relevés itinérants.

L'inventaire floristique a utilisé la
méthode $\quad$ stigmatiste
d'analyse phytosociologique (Braun-Blanquet, 1932), caractérisée par des observations menées à l'intérieur des placeaux. Toutes les espèces rencontrées dans le placeau sont identifiées et répertoriées. Le diamètre du tronc de l'arbre à hauteur de poitrine d'homme a été mesuré pour les individus de $\mathrm{dbh} \geq 10 \mathrm{~cm}$. Des variables écologiques liées à l'influence humaine et aux types de sol ont été enregistrées.

La matrice, espèces $\mathrm{x}$ relevés (présence/absence), a été soumise à la «Detrented Correspondence Analysis (DCA)» et à la classification hiérarchique à l'aide du logiciel Community Analysis Package (CAP). La DCA est une forme améliorée de l'Analyse Factorielle des Correspondances (AFC) qui a permis l'ordination, dans un espace ou plan factoriel les relevés en liaison avec les facteurs écologiques déterminants. Les groupements végétaux ont été individualisés et caractérisés.

La diversité floristique est évaluée à l'aide de la richesse spécifique, du nombre de familles, de l'indice de Shannon et de l'équitabilité de Pielou. La richesse spécifique est le nombre d'espèces présentes 
soit dans un placeau (richesse spécifique moyenne), soit dans la communauté (richesse spécifique totale). La nomenclature botanique utilisée est celle de la Flore Analytique du Bénin (Akoègninou et al., 2006).

L'indice de Shannon $(\mathrm{H})$ utilisé dans le cas des ligneux de diamètre à hauteur de poitrine d'homme d'au moins $10 \mathrm{~cm}$ est donné par la formule suivante :

$\mathrm{H}=-\sum \mathrm{Ni} / \mathrm{Nlog}_{2} \mathbf{N i} / \mathbf{N}$,

$\mathrm{Ni}=$ l'effectif de l'espèce $\mathrm{i}$ et $\mathrm{N}=1$ 'effectif total des espèces (Légendre et Légendre, 1984 ; Frontier et Pichod-Vitale, 1995).

L'équitabilité de Pielou (R), qui détermine le niveau d'organisation du peuplement, rapport entre la diversité observée $(\mathrm{H})$ et la diversité maximale $(\mathrm{S})$, est donnée par :

\section{$\mathbf{R}=\mathbf{H} / \log _{2} \mathrm{~S}$}

Pour Auclaire et Goff (1971), c'est l'équitabilité de Pielou qui permet des comparaisons plus rigoureuses des diversités entre peuplements.

La diversité des familles est analysée pour l'ensemble de la communauté ; c'est le nombre de familles recensées.

Les spectres écologiques ont été construits sur la base des types biologiques de Raunkiaer (1934), de Adjanohoun (1964) et de Guillaumet (1967) et des types phytogéographiques utilisant les subdivisions chorologiques de White (1986) généralement admises pour l'Afrique.

\section{RESULTATS}

\section{Cortège floristique}

Nous avons recensé 123 espèces réparties en espèces autochtones et en espèces introduites (Tableau 1). Les espèces introduites sont : Hevea brasiliensis, Tectona grandis et Erythrophleum suaveolens. La forêt classée de Sakété a connu en effet des travaux d'enrichissement. Parmi les espèces autochtones, les plus fréquentes sont: Anthonotha macrophylla, Cleistopholis patens, Symphonia globulifera, Carapa procera et Ficus vogeliana.

Les espèces recensées appartiennent à 95 genres et 41 familles. Dans la forêt de
Sakété, les familles les mieux représentées sont les Euphorbiaceae et les Leguminosae (16 espèces chacune, soit 13\%), Rubiaceae (8 genres, soit $8,4 \%$ et 13 espèces, soit $10,6 \%$ ), les Apocynaceae ( 6 genres et 6 espèces) et les Moraceae (4 genres et 6 espèces). Ces cinq familles $(12,2 \%)$ sont représentées par 40 genres $(42,1 \%)$ et 57 espèces $(46,3 \%)$.

Les microphanérophytes représentent le type biologique le plus riche en espèces (55). Ils sont suivis des mésophanérophytes (35 espèces), des nanophanérophytes (26 espèces). Les mégaphanérophytes (7 espèces) sont les moins représentés.

S'agissant de la répartition phytogéographiques des espèces, la flore guinéo-congolaise a été la plus riche (97 espèces, soit $78,7 \%$ ) et constitue ainsi l'essentiel du fond floristique de la forêt. Elle est suivie des espèces soudano-guinéennes $(8,1 \%)$, des pantropicales $(4,1 \%)$ et des afrotropicales $(2,4 \%)$. La flore guinéo-orientale, guinéo-occidentale et paléotropicale ne compte chacune que $1,6 \%$ de l'ensemble des espèces. Enfin, la flore afro-américaine et asiatique sont les moins représentées $(0,8 \%$ chacune).

\section{Typologie des formations végétales}

La classification hiérarchique ascendante (Figure 2) et l'ordination des relevés (Figure 3) ont permis de présenter la répartition des placeaux suivant deux axes factoriels principaux.

Trois types de végétation (Figures 2 et 3) ont été identifiés:

- GI ou forêt dense humide semi-décidue à Trilepiseum madagascariensis et Dichapetalum crassifolium rencontré sur des sols de type ferralitique de pentes ;

- GII ou forêt inondable à Anthonotha crassifolia et Symphonia globulifera, rencontré dans la vallée où le sol est de type sablo-argileux à engorgement temporaire ;

- GIII ou forêt marécageuse à Anthostema aubryanum et Alchornea cordifolia, établi sur les sols sableux ou argilo-sableux et vaseux à hydromorphie permanente. 
L'axe 1 de la Figure 3 porte à son origine les relevés de sol marécageux et à son extrémité les relevés de sols exondés. Cet axe peut être interprété comme représentant un gradient d'humidité édaphique liée à la topographie.

\section{Richesse spécifique des formations végétales}

La répartition de la composition floristique dans les trois formations végétales est présentée dans le Tableau 2 qui indique : 54 espèces dans les îlots de la forêt dense semi-décidue (GI), 58 espèces dans la forêt inondable (GII) et 62 espèces dans la forêt marécageuse (GIII).

Le fond floristique est constitué de 8 espèces communes aux trois types de formations végétales. Les espèces différentielles, qui ne sont rencontrées que dans un type de formation végétale: 27 espèces dans la forêt marécageuse, 27 espèces dans la forêt dense semi-décidue et 20 espèces dans la forêt inondable.

Le Tableau 2 présente la variation de la richesse spécifique en fonction des familles pour l'ensemble des trois formations végétales. Les Leguminosae constituent la famille la plus diversifiée en forêt dense humide semi-décidue ( 7 genres et 9 espèces) et dans la forêt inondable ( 7 genres et 8 espèces). Les Rubiaceae viennent en deuxième position dans la forêt dense humide semi-décidue (5 genres monospécifiques) et dans la forêt inondable (4 genres et 7 espèces). Par contre, les Euphorbiaceae représentent la famille la plus riche en genres (9) et en espèces (11) au niveau de la forêt marécageuse.

\section{Spectres écologiques des formations végétales \\ La Figure 4 présente le spectre biologique des formations végétales. Elle montre que les microphanérophytes sont les plus riches en espèces dans les îlots de forêt dense humide semi-décidue avec un taux de $53,7 \%$ et dans la forêt inondable $(43,1 \%)$. Par}

contre, les mésophanérophytes prédominent en forêt marécageuse $(43,6 \%)$.

La flore guinéo-congolaise est la plus diversifiée au sein des trois groupements végétaux où leur taux est compris entre 75,9\% en forêt dense humide semi-décidue et $87,9 \%$ en forêt inondable (Figure 5). Les autres types phytogéographiques sont partout faiblement représentés.

\section{Structure des formations végétales}

La structure verticale de la forêt dense humide semi-décidue à Trilepiseum madagascariensis et Dichapetalum crassifolium est essentiellement constituée de :

- une strate arborée de 20 à 25 m de hauteur. Les espèces les plus abondantes sont celles de Trilepiseum madagascariensis et Erythrophleum suaveolens ;

- une strate arbustive, de 6 à 15 m de hauteur, représentée par des espèces dont les plus abondantes sont Drypetes floribunda et Dichapetalum crassifolium ;

- une strate sous-arbustive, de 1 à $3 \mathrm{~m}$ de hauteur, constituée des espèces comme Reissantia indica, Uvaria chamae et Mallotus oppositifolius.

Le peuplement ligneux $(\mathrm{dbh} \geq 10 \mathrm{~cm})$ compte 10 espèces, avec un indice de diversité de Shannon de 2,4 bits et une équitabilité de Pielou de 0,5. La densité des arbres de dbh $\geq$ $10 \mathrm{~cm}$ est de 200 tiges/ha. La surface terrière est de 20,2 $\mathrm{m}^{2} / \mathrm{ha}$. Le diamètre de l'arbre moyen est de $35,9 \mathrm{~cm}$. Les individus les plus nombreux sont ceux de faible diamètre (Figure 6a).

La forêt inondable à Anthonotha crassifolia et Symphonia globulifera est constituée de :

- une strate arborescente de 25 à $30 \mathrm{~m}$ de hauteur. On y rencontre des espèces telles que Anthonotha crassifolia, Zanthoxylum leprieurii, Anthonotha macrophylla et Symphonia globulifera;

- une strate arbustive de 10 à $15 \mathrm{~m}$ de hauteur et dont le fond floristique est essentiellement composé de Raphia hookeri, Olax subscorpioidea et Psychotria articulata ; 
- une strate sous-arbustive, de 1 à $3 \mathrm{~m}$ de hauteur, dominée par Trichilia monodelpha et Cremaspora triflora.

Le peuplement arborescent $(\mathrm{dbh} \geq 10$ $\mathrm{cm})$ compte 12 espèces, avec un indice de Shannon de 3,4 bits et une équitabilité de Pielou de 0,8. La densité des arbres de dbh $\geq$ $10 \mathrm{~cm}$ de cette phytocénose est de 80 tiges /ha. La surface terrière est de $13 \mathrm{~m}^{2} / \mathrm{ha}$. Le diamètre de l'arbre moyen est de $45,4 \mathrm{~cm}$. Les individus les plus nombreux sont ceux ayant $25 \mathrm{~cm}$ pour centre de classes (Figure $6 \mathrm{~b}$ ).

La forêt marécageuse à Anthostema aubryanum et Alchornea cordifolia présente : - une strate arborescente, de 20 à $25 \mathrm{~m}$ de hauteur, composée d'arbres à frondaison discontinue tels que Anthostema aubryanum, Hevea brasiliensis, Anthonotha macrophylla, Ceiba pentandra et Cleistopholis patens ;
- la strate arbustive, de 5 à $15 \mathrm{~m}$ de hauteur, où l'on observe Symphonia globulifera, Musanga cecropioides, Alstonia congensis, Carapa procera, Ficus vogeliana, Funtumia africana, etc. ;

- la strate sous-arbustive de 1 à $3 \mathrm{~m}$ de hauteur, est constituée de jeunes individus d'espèces des deux strates précédentes.

Le peuplement arborescent $(\mathrm{dbh} \geq 10$ $\mathrm{cm})$ renferme 22 espèces avec un indice de Shannon de 3,8 bits et une équitabilité de Pielou de 0,6. La densité des arbres de dbh $\geq$ $10 \mathrm{~cm}$ est de 137 tiges/ha pour cette phytocénose. La surface terrière est de 18,5 $\mathrm{m}^{2} /$ ha. Le diamètre de l'arbre moyen est de $34,1 \mathrm{~cm}$. Les individus les plus nombreux sont ceux de faible diamètre (Figure $6 \mathrm{c}$ ).

Tableau 1 : Liste des espèces ligneuses recensées dans la forêt classée de Sakété.

\begin{tabular}{|c|c|c|c|c|c|c|}
\hline TB & TP & Espèces & Familles & FDHS & FI & FM \\
\hline Lmph & At & Acacia erythrocalyx Brenan. & Leguminosae & & 1 & \\
\hline Lmph & GC & Acridocarpus alternifolius (Schum \& Thonn.) Nied. & Malpighiaceae & & 1 & \\
\hline Lnph & GC & Agelaea pentagyna (Lam.) Baill. & Connaraceae & & & 1 \\
\hline $\mathrm{mPh}$ & GC & Albizia adianthifolia (Schum.) W.Wight & Leguminosae & 1 & & \\
\hline $\mathrm{mPh}$ & GC & Alstonia congensis Engl. & Apocynaceae & & & 1 \\
\hline nph & GC & Angylocalyx oligophyllus (Baker) Baker & Leguminosae & 1 & 1 & 1 \\
\hline $\mathrm{mPh}$ & GC & Anthocleista vogelii Planch. & Loganiaceae & & 1 & 1 \\
\hline $\mathrm{mPh}$ & GC & Anthonotha crassifolia (Baill .) J. Leonard & Leguminosae & & 1 & 1 \\
\hline $\mathrm{mPh}$ & GC & Anthonotha macrophylla P.Beauv. & Leguminosae & & 1 & 1 \\
\hline $\mathrm{mPh}$ & GC & Anthostema aubryanum Baill. & Euphorbiaceae & & & 1 \\
\hline $\mathrm{MPh}$ & GC & Antiaris toxicaria Lesch. & Moraceae & 1 & & \\
\hline $\mathrm{mph}$ & GC & Antidesma laciniatum Müll. Arg. subsp. laciniatum & Euphorbiaceae & & 1 & \\
\hline $\mathrm{mph}$ & GC & Antidesma membranaceum Müll. Arg. & Euphorbiaceae & & & 1 \\
\hline $\mathrm{mPh}$ & $\mathrm{Pt}$ & Artocarpus altilis (Parkinson) Fosberg & Moraceae & & & 1 \\
\hline $\mathrm{mph}$ & GC & Baphia nitida Lodd. & Leguminosae & 1 & 1 & 1 \\
\hline $\mathrm{mph}$ & GC & Baphia pubescens Hook.f. & Leguminosae & 1 & & \\
\hline $\mathrm{mPh}$ & $\mathrm{Pt}$ & Blighia sapinda Koenig & Sapindaceae & 1 & & \\
\hline $\mathrm{mPh}$ & GC & Bridelia grandis Pierre ex Hutch. & Euphorbiaceae & & & 1 \\
\hline $\mathrm{mPh}$ & At & Bridelia micrantha (Hochst.) Baill. & Euphorbiaceae & & & 1 \\
\hline nph & GC & Campylospermum glaberrimum (P. Beauv.) Farron & Ochnaceae & & 1 & \\
\hline
\end{tabular}




\begin{tabular}{|c|c|c|c|c|c|c|}
\hline Lmph & $\mathrm{GC}$ & Campylostemon warneckeanum Loes. ex Fritsch & Celastraceae & 1 & 1 & \\
\hline nph & GC & Capparis erythrocarpos Isert & Capparidaceae & 1 & & \\
\hline $\mathrm{mPh}$ & GC & Carapa procera DC. & Meliaceae & & & 1 \\
\hline $\mathrm{mph}$ & GC & Carpolobia lutea G.Don & Polygalaceae & 1 & 1 & 1 \\
\hline $\mathrm{MPh}$ & $\mathrm{Pt}$ & Ceiba pentandra (L.) Gaertn. & Bombacaceae & & & 1 \\
\hline $\mathrm{mPh}$ & GC & Celtis mildbraedii Engl. & Celtidaceae & 1 & & \\
\hline $\mathrm{MPh}$ & GC & Celtis zenkeri Engl. & Celtidaceae & & & 1 \\
\hline $\mathrm{nph}$ & GC & Chassalia kolly (Schumach.) Hepper & Rubiaceae & & 1 & 1 \\
\hline $\mathrm{mPh}$ & GC & Cleistopholis patens (Benth.) Engl. \& Diels & Annonaceae & & 1 & 1 \\
\hline Lmph & $\mathrm{GC}$ & Cnestis corniculata Lam. & Connaraceae & 1 & 1 & 1 \\
\hline nph & GC & Cnestis ferruginea Vahl ex DC. & Connaraceae & 1 & 1 & \\
\hline $\mathrm{mPh}$ & GC & Cola gigantea A. Chev. & Sterculiaceae & 1 & & 1 \\
\hline $\mathrm{mph}$ & GC & Cola millenii K. Schum. & Sterculiaceae & 1 & 1 & 1 \\
\hline Lnph & SG & Combretum paniculatum Vent. & Combretaceae & 1 & & \\
\hline Lmph & $\mathrm{GC}$ & Combretum racemosum P.Beauv. & Combretaceae & 1 & & \\
\hline Lmph & $\mathrm{GC}$ & Connarus thonningii & Connaraceae & 1 & 1 & 1 \\
\hline $\mathrm{nph}$ & At & Cremaspora triflora (Thonn.) K.Schum. & Rubiaceae & 1 & 1 & \\
\hline $\mathrm{LmPh}$ & $\mathrm{GC}$ & Dalbergia afzeliana G. Don & Leguminosae & & 1 & 1 \\
\hline Lmph & GE & Dalbergia lactea Vatke & Leguminosae & & & 1 \\
\hline $\mathrm{mph}$ & GO & Dalbergia setifera Hutch.\& Dalziel & Leguminosae & 1 & & \\
\hline $\mathrm{mph}$ & $\mathrm{Pt}$ & Dalbergia sissoo Roxb. ex DC. & Leguminosae & 1 & & \\
\hline MPh & GC & Daniellia ogea (Harms) Rolfe ex Holland. & Leguminosae & & 1 & 1 \\
\hline nph & GC & Deinbollia pinnata (Poir.) Schumach. \& Thonn. & Sapindaceae & 1 & & \\
\hline $\mathrm{mPh}$ & GC & Dialium guineense Willd. & Leguminosae & 1 & & \\
\hline Lmph & $\mathrm{GC}$ & Dichapetalum crassifolium Chodat, & Dichapetalaceae & 1 & & \\
\hline $\mathrm{mph}$ & GC & Dichapetalum madagascariense Poir. & Dichapetalaceae & 1 & & \\
\hline $\mathrm{mph}$ & GC & Diospyros monbuttensis Gürke & Ebenaceae & & & 1 \\
\hline $\mathrm{mph}$ & GC & Drypetes floribunda (Müll. Arg.) Hutch. & Euphorbiaceae & 1 & 1 & \\
\hline nph & GC & Drypetes gilgiana $(\mathrm{Pax})$ Pax \& Hoffm. & Euphorbiaceae & & 1 & \\
\hline $\mathrm{mph}$ & GC & Drypetes leonensis Pax & Euphorbiaceae & & & 1 \\
\hline $\mathrm{mPh}$ & SG & Erythrophleum suaveolens (Guill. \& Pierr.) Brenan & Leguminosae & 1 & & \\
\hline $\mathrm{mph}$ & GC & Ficus lutea Vahl & Moraceae & & & 1 \\
\hline $\mathrm{mph}$ & GC & Ficus lyrata Warb. & Moraceae & & 1 & \\
\hline $\mathrm{mPh}$ & GC & Ficus vogeliana (Miq.) Miq. & Moraceae & & & 1 \\
\hline $\mathrm{mPh}$ & GC & Funtumia africana (Benth.) Stapf & Apocynaceae & & 1 & 1 \\
\hline $\mathrm{mph}$ & GC & Glyphaea brevis (Spreng.) Monachino & Tiliaceae & & & 1 \\
\hline $\mathrm{mPh}$ & GC & Hannoa klaineana Pierre \& Engl. & Simaroubaceae & & & 1 \\
\hline $\mathrm{mph}$ & SG & Harissonia abyssinica Oliv. & Simaroubaceae & 1 & & \\
\hline $\mathrm{mph}$ & Pal & Harungana madagascariensis Lam. ex Poir. & Clusiaceae & & & 1 \\
\hline
\end{tabular}




\begin{tabular}{|c|c|c|c|c|c|c|}
\hline $\mathrm{mPh}$ & $\mathrm{A}$ & Hevea brasiliensis (Kunth) Müll. Arg & Euphorbiaceae & & & 1 \\
\hline $\mathrm{mPh}$ & GC & Homalium stipulaceum Welw. Ex Mast. & Flacourtiaceae & & & 1 \\
\hline $\mathrm{mPh}$ & Am & Hura crepitans $\mathrm{L}$. & Euphorbiaceae & 1 & & \\
\hline Lnph & GE & Icacina trichantha Oliv. & Icacinaceae & 1 & 1 & \\
\hline $\mathrm{MPh}$ & $\mathrm{GC}$ & $\begin{array}{l}\text { Irvingia gabonensis (Aubry-Leconte ex O'Roche) } \\
\text { Baill. }\end{array}$ & Irvingiaceae & & 1 & \\
\hline Lmph & GC & Keetia hispida (Benth.) Bridson & Rubiaceae & 1 & & \\
\hline $\mathrm{mph}$ & GC & Lecaniodiscus cupanioides Planch. & Sapindaceae & 1 & 1 & \\
\hline $\mathrm{mph}$ & $\mathrm{GC}$ & Macaranga barteri Müll. Arg & Euphorbiaceae & & & 1 \\
\hline $\mathrm{mph}$ & GC & Macaranga heterophylla Müll. Arg & Euphorbiaceae & & & 1 \\
\hline $\mathrm{mph}$ & GO & Maesobotrya barteri (Baill.) Hutch. & Euphorbiaceae & & & 1 \\
\hline nph & Pal & Mallotus oppositifolius (Geisel.) Müll. Arg & Euphorbiaceae & 1 & 1 & \\
\hline $\mathrm{MPh}$ & GC & Maranthes robusta (Oliv.) Prance ex F. White & Chrysobalanaceae & & 1 & \\
\hline Lnph & GC & Memecylon afzelii G. Don & Melastomataceae & 1 & & \\
\hline $\mathrm{mPh}$ & $\mathrm{GC}$ & Monodora myristica (Gaertn.) Dunal & Annonaceae & & & 1 \\
\hline $\mathrm{mph}$ & GC & Monodora tenuifolia Benth. & Annonaceae & 1 & 1 & \\
\hline $\mathrm{mPh}$ & $\mathrm{GC}$ & Musanga cecropioides R.Br. & Cecropiaceae & & 1 & 1 \\
\hline $\mathrm{mph}$ & $\mathrm{GC}$ & Newbouldia laevis (P. Beauv.) Seem. ex Bureau & Bignoniaceae & & 1 & \\
\hline nph & $\mathrm{GC}$ & Olax gambecola Baill. & Olacaceae & & 1 & 1 \\
\hline $\mathrm{mph}$ & GC & Olax subscorpioidea Oliv. & Olacaceae & 1 & 1 & \\
\hline nph & GC & Oxyanthus racemosus (Schum. \& Thonn.) Keay & Rubiaceae & & & 1 \\
\hline $\mathrm{mph}$ & GC & Oxyanthus unilocularis Hiern & Rubiaceae & 1 & & 1 \\
\hline $\mathrm{mph}$ & $\mathrm{GC}$ & Pancovia bijuga Willd. & Sapindaceae & & 1 & \\
\hline $\mathrm{mph}$ & SG & Pandanus candelabrum P. Beauv. & Pandanaceae & & 1 & \\
\hline $\mathrm{mPh}$ & $\mathrm{GC}$ & Parkia bicolor A. Chev. & Leguminosae & & 1 & 1 \\
\hline $\mathrm{mph}$ & SG & Pavetta corymbosa (DC.) F. . Williams & Rubiaceae & 1 & & \\
\hline Lmph & $\mathrm{GC}$ & $\begin{array}{l}\text { Philenoptera cyanescens (Schumach. \& Thonn.) } \\
\text { Roberty }\end{array}$ & Leguminosae & 1 & & \\
\hline nph & $\mathrm{GC}$ & Pleiocarpa pycnantha (K. Schum.) Stapf & Apocynaceae & 1 & 1 & 1 \\
\hline $\mathrm{mph}$ & GC & Pouteria alnifolia (Baker) Roberty & Sapotaceae & 1 & & \\
\hline $\mathrm{mph}$ & $\mathrm{GC}$ & Psychotria articulata (Hiern) Petit & Rubiaceae & & 1 & \\
\hline nph & GC & Psychotria calva Hiern & Rubiaceae & & 1 & 1 \\
\hline nph & SG & Psychotria psychotrioides (DC.) Roberty & Rubiaceae & & 1 & \\
\hline nph & SG & Psychotria vogeliana Benth. & Rubiaceae & & & 1 \\
\hline $\mathrm{mPh}$ & GC & Pycnanthus angolensis (Welw.) Warb. & Myristicaceae & 1 & 1 & 1 \\
\hline Lmph & $\mathrm{GC}$ & Pyrenacantha vogeliana Baill. & Icacinaceae & 1 & & 1 \\
\hline $\mathrm{mph}$ & $\mathrm{GC}$ & Raphia hookeri G.Mann \& H.Wendl. & Arecaceae & & 1 & 1 \\
\hline mph & SG & Rauvolfia vomitoria Afzel. & Apocynaceae & 1 & & 1 \\
\hline Lnph & $\mathrm{GC}$ & Reissantia indica (Willd.) N. Hallé & Celastraceae & 1 & & \\
\hline Lnph & GC & $\begin{array}{l}\text { Rhaphiostylis beninense (Hook.f. ex Planch.) Planch. } \\
\text { ex Benth. }\end{array}$ & Icacinaceae & 1 & 1 & \\
\hline
\end{tabular}




\begin{tabular}{|c|c|c|c|c|c|c|}
\hline nph & GC & Rinorea batesii Chipp. & Violaceae & & 1 & \\
\hline $\mathrm{mph}$ & GC & Rinorea dentata (P. Beauv.) O.Ktze. & Violaceae & 1 & & \\
\hline nph & GC & Rinorea kibbiensis Chipp. & Violaceae & & & 1 \\
\hline $\mathrm{mph}$ & GC & Rothmannia longiflora Salisb & Rubiaceae & 1 & & \\
\hline Lmph & GC & Rutidea parviflora DC. & Rubiaceae & & 1 & \\
\hline Lmph & $\mathrm{GC}$ & Rutidea smithii Hiern & Rubiaceae & & 1 & \\
\hline $\mathrm{nph}$ & $\mathrm{GC}$ & Salacia pallescens Oliv. & Celastraceae & & 1 & \\
\hline Lmph & GC & Sorindeia grandifolia Engl. & Anacardiaceae & 1 & 1 & \\
\hline nph & GC & Sphenocentrum jollyanum Pierre & Menispermaceae & 1 & 1 & \\
\hline $\mathrm{mPh}$ & GC & Spondianthus preussii Engl. var. preussii & Euphorbiaceae & & 1 & 1 \\
\hline $\mathrm{mPh}$ & GC & Sterculia tragacantha Lindl. & Sterculiaceae & & 1 & 1 \\
\hline $\mathrm{mPh}$ & GC & Symphonia globulifera L.f. & Clusiaceae & & 1 & 1 \\
\hline Lnph & $\mathrm{GC}$ & Tabernaemontana pachysiphon Stapf & Apocynaceae & & & 1 \\
\hline $\mathrm{mPh}$ & $\mathrm{Pt}$ & Tectona grandis L.f. & Verbenaceae & 1 & & \\
\hline $\mathrm{mPh}$ & $\mathrm{GC}$ & Trichilia monadelpha (Thonn.) J.J. de Wilde & Meliaceae & & 1 & \\
\hline $\mathrm{mph}$ & GC & Trichilia prieuriana A. Juss. subsp. & Meliaceae & 1 & & \\
\hline $\mathrm{mPh}$ & GC & Trichilia tessmannii Harms. & Meliaceae & & & 1 \\
\hline $\mathrm{mPh}$ & GC & Trilepisium madagascariensis DC. & Moraceae & 1 & & 1 \\
\hline MPh & GC & Triplochiton scleroxylon K. Schum. & Sterculiaceae & 1 & & \\
\hline $\mathrm{mPh}$ & GC & Uapaca paludosa Aubrev. \& Léandri & Euphorbiaceae & & 1 & 1 \\
\hline Lmph & GC & Uvaria chamae P. Beauv. & Annonaceae & 1 & & \\
\hline $\mathrm{mph}$ & GC & Uvaria doeringii Diels & Annonaceae & & & 1 \\
\hline $\mathrm{mph}$ & GC & Vitex grandifolia Gürke & Verbenaceae & & 1 & 1 \\
\hline $\mathrm{mph}$ & SG & Voacanga africana Stapf & Apocynaceae & & 1 & \\
\hline $\mathrm{mph}$ & SG & Xylopia parviflora (A.Rich.) Benth. & Annonaceae & & & 1 \\
\hline $\mathrm{mph}$ & GC & Zanthoxylum leprieurii Guill. \& Perr. & Rutaceae & & 1 & \\
\hline
\end{tabular}


Tableau 2: Variation de la richesse spécifique et du nombre de genres au sein des familles importantes.

\begin{tabular}{|c|c|c|c|c|c|c|}
\hline \multirow[t]{2}{*}{ Familles } & \multicolumn{2}{|c|}{ FDHSD } & \multicolumn{2}{|c|}{ FI } & \multicolumn{2}{|c|}{ FM } \\
\hline & Genre & Espèce & Genre & Espèce & Genre & Espèce \\
\hline Anacardiaceae & 1 & 1 & 1 & 1 & & \\
\hline Annonaceae & 2 & 2 & 2 & 2 & 4 & 4 \\
\hline Apocynaceae & 2 & 2 & 3 & 3 & 5 & 5 \\
\hline Arecaceae & & & 1 & 1 & 1 & 1 \\
\hline Bignoniaceae & & & 1 & 1 & & \\
\hline Bombacaceae & & & & & 1 & 1 \\
\hline Capparidaceae & 1 & 1 & & & & \\
\hline Cecropiaceae & & & 1 & 1 & 1 & 1 \\
\hline Celastraceae & 2 & 2 & 2 & 2 & & \\
\hline Celtidaceae & 1 & 1 & & & 1 & 1 \\
\hline Chrysobalanaceae & & & 1 & 1 & & \\
\hline Clusiaceae & & & 1 & 1 & 2 & 2 \\
\hline Combretaceae & 1 & 2 & & & & \\
\hline Connaraceae & 2 & 3 & 2 & 3 & 3 & 3 \\
\hline Dichapetalaceae & 1 & 2 & & & & \\
\hline Ebenaceae & & & & & 1 & 1 \\
\hline Euphorbiaceae & 3 & 3 & 5 & 6 & 9 & 11 \\
\hline Flacourtiaceae & & & & & 1 & 1 \\
\hline Icacinaceae & 3 & 3 & 2 & 2 & 1 & 1 \\
\hline Irvingiaceae & & & 1 & 1 & & \\
\hline Leguminosae & 7 & 9 & 7 & 8 & 6 & 8 \\
\hline Loganiaceae & & & 1 & 1 & 1 & 1 \\
\hline Malpighiaceae & & & 1 & 1 & & \\
\hline Melastomataceae & 1 & 1 & & & & \\
\hline Meliaceae & 1 & 1 & 1 & 1 & 2 & 2 \\
\hline Menispermaceae & 1 & 1 & 1 & 1 & & \\
\hline Moraceae & 2 & 2 & 1 & 1 & 3 & 5 \\
\hline Myristicaceae & 1 & 1 & 1 & 1 & 1 & 1 \\
\hline Ochnaceae & & & 1 & 1 & & \\
\hline Olacaceae & 1 & 1 & 1 & 2 & 1 & 1 \\
\hline Pandanaceae & & & 1 & 1 & & \\
\hline Polygalaceae & 1 & 1 & 1 & 1 & 1 & 1 \\
\hline Rubiaceae & 5 & 5 & 4 & 7 & 3 & 5 \\
\hline Rutaceae & & & 1 & 1 & & \\
\hline Sapindaceae & 3 & 3 & 2 & 2 & & \\
\hline Sapotaceae & 1 & 1 & & & & \\
\hline Simaroubaceae & 1 & 1 & & & 1 & 1 \\
\hline Sterculiaceae & 2 & 3 & 2 & 2 & 2 & 3 \\
\hline Tiliaceae & & & & & 1 & 1 \\
\hline Verbenaceae & 1 & 1 & 1 & 1 & 1 & 1 \\
\hline Violaceae & 1 & 1 & 1 & 1 & 1 & 1 \\
\hline Total & 48 & 54 & 51 & 58 & 54 & 62 \\
\hline
\end{tabular}




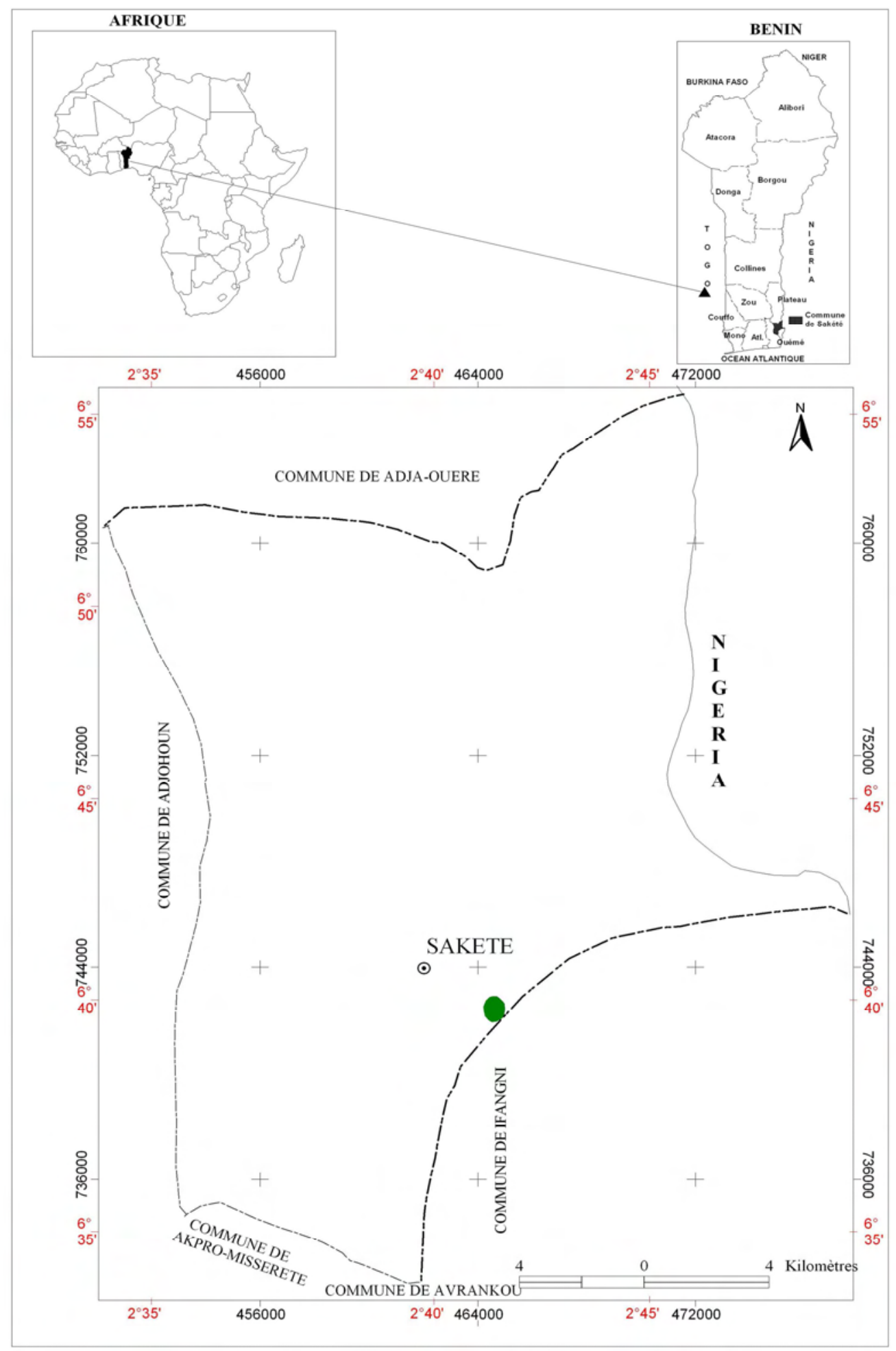

Figure 1 : Localisation géographique de la zone d'étude. 


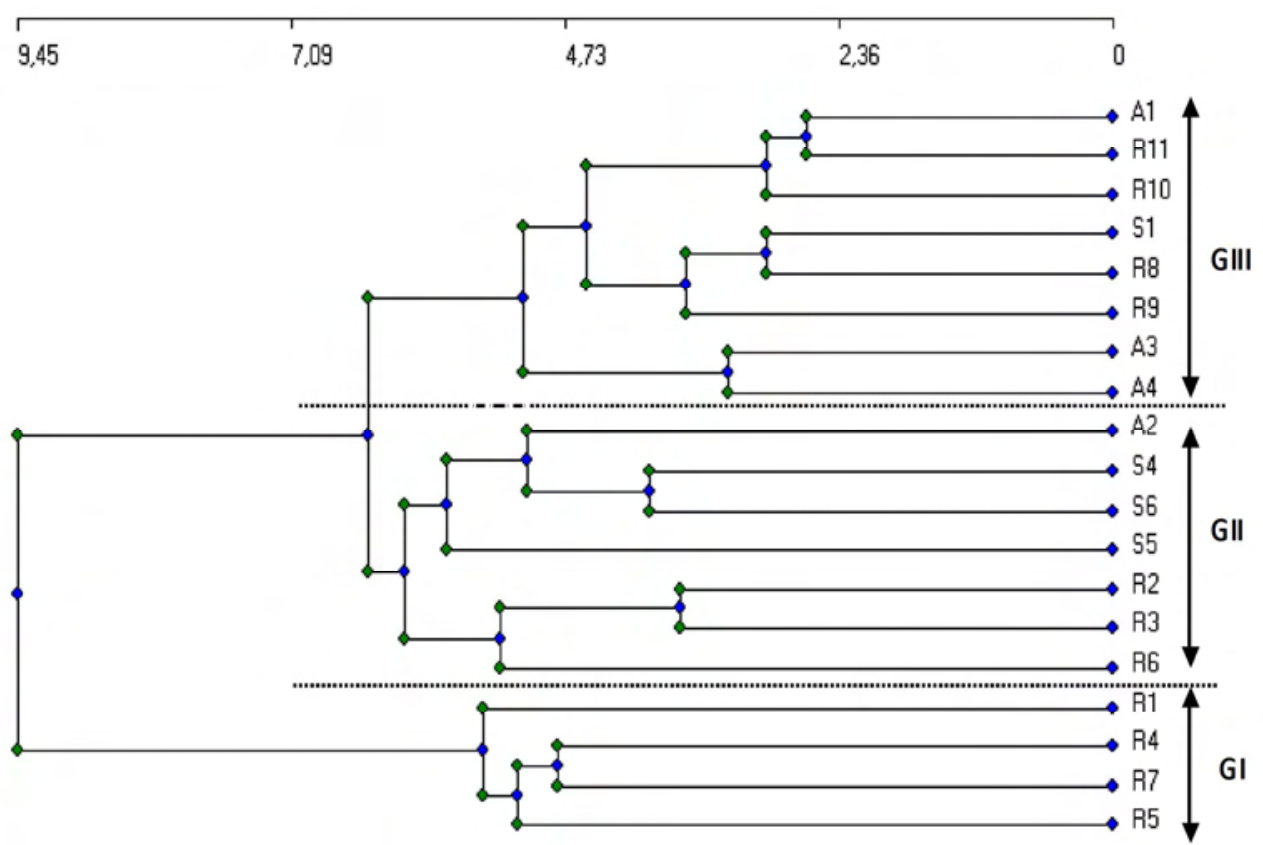

Figure 2 : Dendrogramme des groupes de relevés.

(GI : groupe de placeaux à Trilepiseum madagascariensis et Dichapetalum crassifolium, GII : groupe de placeaux à Anthonotha crassifolia et Symphonia globulifera, GIII : groupe de placeaux à Anthostema aubryanum et Alchornea cordifolia).

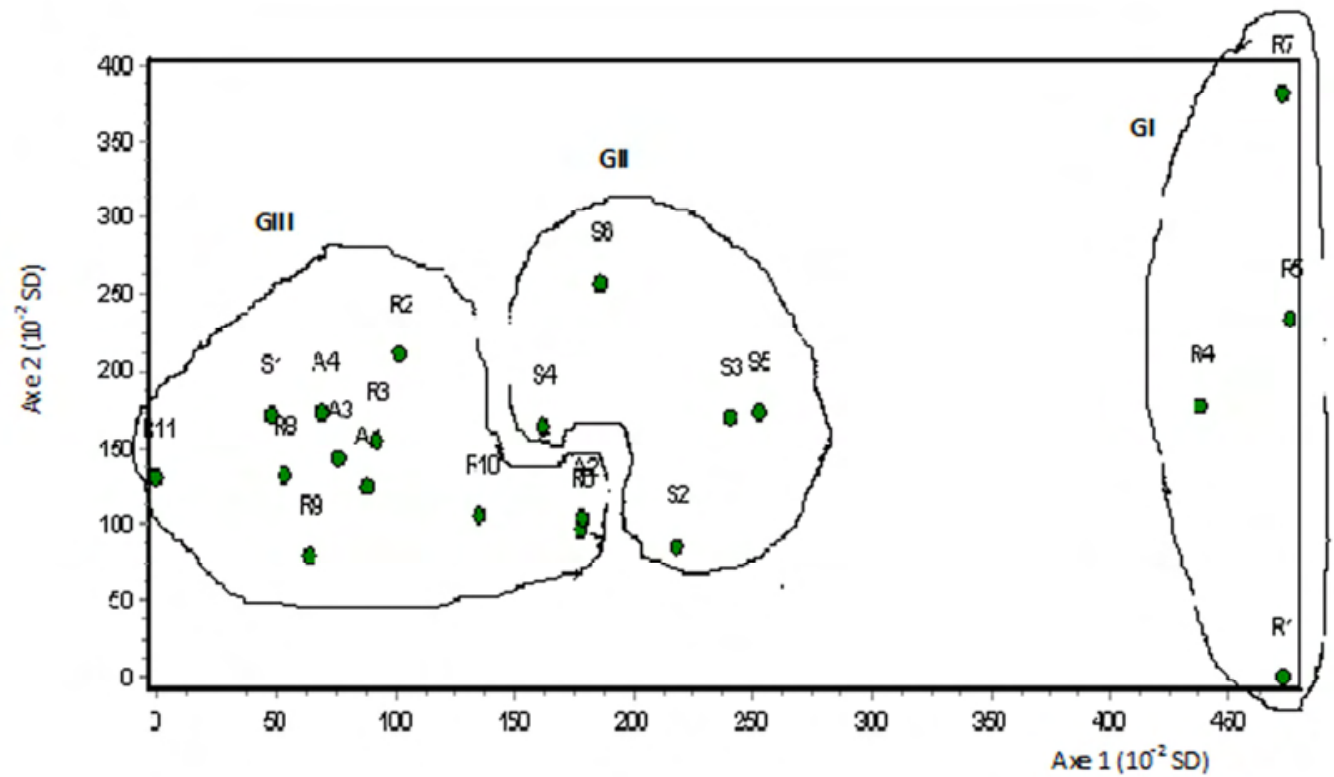

Figure 3 : Individualisation des formations végétales.

(GI : îlot de forêt dense humide semi-décidue de Trilepiseum madagascariensis et Dichapetalum crassifolium, GII : îlot de forêt inondable à Anthonotha crassifolia et Symphonia globulifera, GIII : îlot de forêt marécageuse à Anthostema aubryanum et Alchornea cordifolia). 


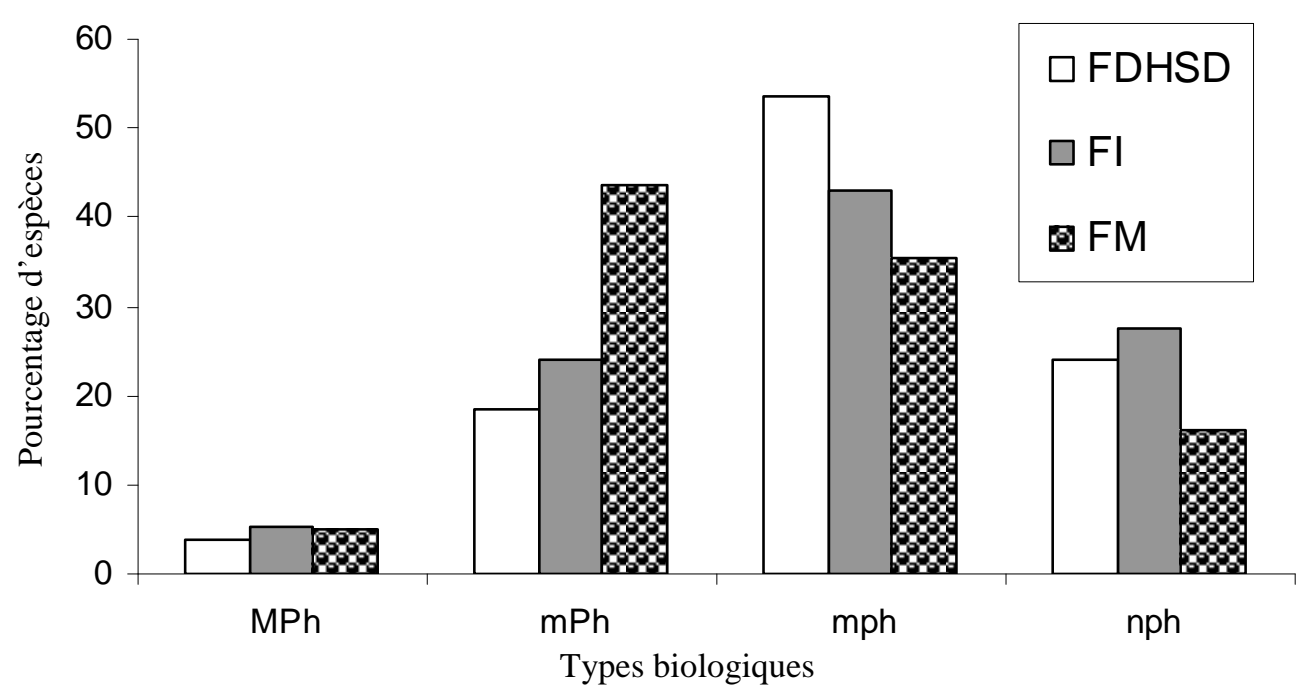

Figure 4 : Spectre biologique des formations végétales.

(MPh : mégaphanérophytes, $\mathrm{mPh}$ : mésophanérophytes, mph : microphanérophytes, nph : nanophanérophytes, FDHSD : îlot de forêt dense humide semi-décidue de Trilepiseum madagascariensis et Dichapetalum crassifolium, FI : îlot de forêt inondable à Anthonotha crassifolia et Symphonia globulifera, FMI : îlot de forêt marécageuse à Anthostema aubryanum et Alchornea cordifolia).

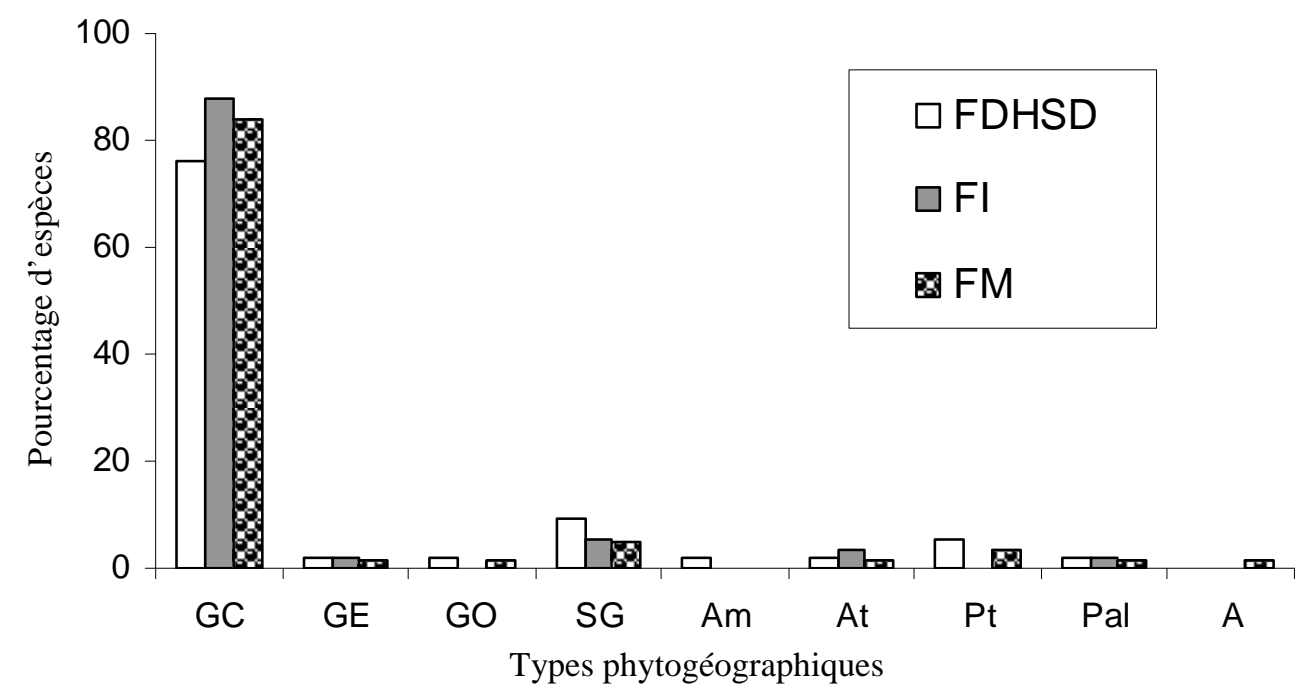

Figure 5 : Spectre phytogéographique des formations végétales.

(GC : guinéo-congolaises, GE : guinéo-orientales, GO : guinéo-occidentales, SG : soudano-guinéennes, Am : afroaméricaines, At : afro-tropicales, Pt : pantropicales, Pal : paléotropicales, A : asiatiques, FDHSD : îlot de forêt dense humide semi-décidue de Trilepiseum madagascariensis et Dichapetalum crassifolium, FI : îlot de forêt inondable à Anthonotha crassifolia et Symphonia globulifera, FMI : îlot de forêt marécageuse à Anthostema aubryanum et Alchornea cordifolia). 

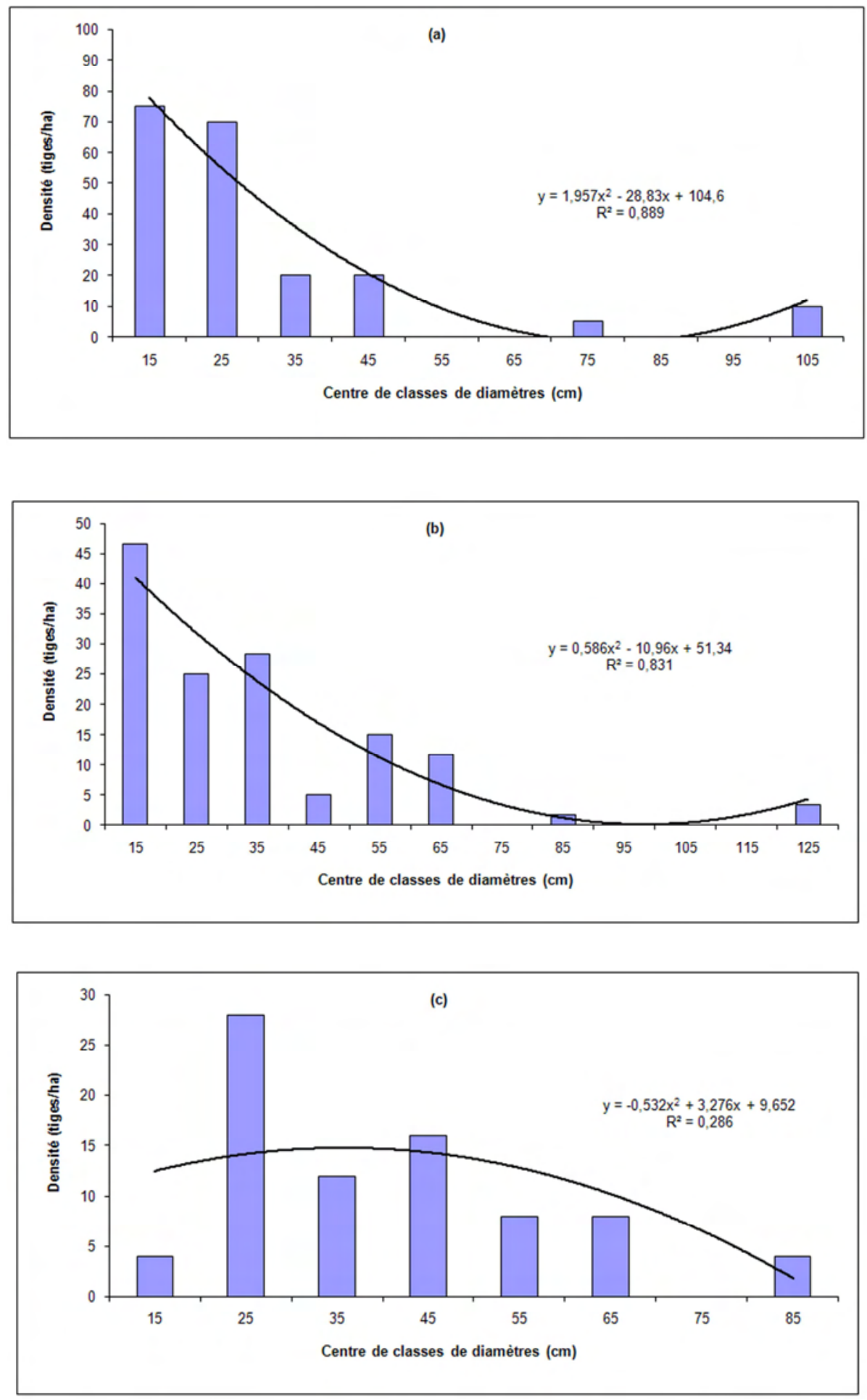

Figure 6 : Répartition par centre des classes de diamètre des arbres. (a : forêt dense humide semi-décidue, $\mathbf{b}$ : forêt marécageuse, $\mathbf{c}$ : forêt inondable). 


\section{DISCUSSION}

Les caractéristiques du peuplement ligneux de la forêt classée de Sakété on été établies en utilisant divers indices de diversité (richesse spécifique, indice de Shannon et équitabilité de Pielou) et des paramètres de structure. Les observations ont consisté en des inventaires floristiques et des relevés de végétation dans des placettes de $500 \mathrm{~m}^{2}$.

La richesse floristique est relativement élevée. Le rapport entre le nombre d'espèces et celui des genres a été de 8,$3 ; 13,7$ et $14,8 \%$ des genres respectivement dans la forêt sèche semi-décidue, la forêt inondable et la forêt marécageuse.

Le nombre de familles au niveau de la forêt étudiée est relativement faible; il est plus faible à ceux rapportés par Sokpon (1995) (71) dans la forêt naturelle de Pobè et Hountondji (1998) (78) dans la forêt de Niaouli.

L'indice de diversité est comparable à celui obtenu dans la forêt classée de la Lama (2,3 à 2,8 bits) et même dans les forêts sacrées du Sud-Bénin (2,7 à 3,8 bits) et du plateau Adja (2,7 à 3,1 bits). Il est en revanche inférieur aux valeurs enregistrées dans les forêts sacrées du Sud-est du Bénin (4,7 à 5,0 bits). Cette différence proviendrait de l'action anthropique.

Les valeurs de l'équitabilité de Pielou ont été de 0,$5 ; 0,6$ et 0,8 respectivement pour la forêt dense humide semi-décidue, la forêt marécageuse et la forêt inondable. La forêt inondable à Anthonotha crassifolia et Symphonia globulifera apparaît comme présentant un bon niveau d'organisation, et ainsi une meilleure stabilité du milieu.

La densité est aussi faible, comparativement à celle observée dans les forêts sacrées du plateau Adja (244 à 335 tiges/ha) ou dans celles du Sud-est du Bénin (312 à 665 tiges/ha) ou dans la forêt classée de la Lama au Sud-Bénin (360 à 442 tiges/ha). Cela traduirait certainement une exploitation intense des ressources ligneuses par les populations riveraines.

La distribution des arbres en fonction des diamètres a été ajustée à différentes lois de type polynomial dont les représentations s'apparentent à la fonction exponentielle. Cette ressemblance proviendrait certainement de deux phénomènes importants. Le premier est la présence des arbres de faible diamètre (jeunes plants) qui rendraient compte du fort potentiel de rajeunissement du peuplement, un peuplement qui régénère. Le second évènement serait la forte exploitation des arbres de gros diamètre, pour la production de bois de feu, de bois de service, ou de bois d'œuvre. Ces phénomènes apparaissent bien dans les surfaces terrières (13 à 20,2 $\mathrm{m}^{2} / \mathrm{ha}$ ), relativement plus faibles que celles obtenues dans les forêts denses semi-décidues du Bénin (27,9 à 50,7 m²/ha).

Pour Slatyer et al. (1977), cités par Agbani (2002), la distribution des arbres par classes de diamètre est la donnée fondamentale pour l'étude statistique des forêts tropicales. Mais une loi de distribution exponentielle ne rend pas suffisamment compte des distributions réelles des arbres par classes de diamètre. Il est en effet important de tenir compte des influences des principaux facteurs du milieu (sol, topographie...) et les phénomènes de compétition pour une meilleure étude de la distribution et de l'architecture des forêts.

\section{Conclusion}

L'état actuel du peuplement ligneux de la forêt classée de Sakété au Sud-Bénin a été établi. La densité des arbres est faible, le niveau d'organisation varie en fonction du milieu, la richesse floristique est relativement élevée. Les fonctions économiques de ce milieu sont en train d'être érodées, en raison des dégâts (coupe anarchique de bois et feux de végétation) causés par les populations riveraines, donc une dégradation de leur cadre de vie. Comme conséquence, la végétation naturelle a régressé au profit des formations anthropiques (plantations, jachères et champs).

Un plan d'aménagement et de gestion participatif en relation avec les populations et les autorités locales et/ou régionales devrait 
permettre de redonner à cet espace ses fonctions écologiques et socio-économiques.

\section{REFERENCES}

Adjanohoun E. 1964. Végétation des Savanes et des Rochers Découverts en Côte d'Ivoire Centrale. ORSTOM.

Agbani P. 2002. Cartographie fonctionnelle par bandes longitudinales à grandes échelles des petits îlots boisés: Cas du noyau central de la forêt classée de la Lama. Mémoire de DEA, Bénin, p. 74.

Akoègninou A. 1984. Contribution à l'étude botanique des îlots de forêts dense humides semi-décidues en République populaire $\mathrm{du}$ Bénin. Thèse $\mathrm{du} 3^{\mathrm{e}}$ cycle, Université de Bordeaux III, p. 250.

Akoègninou A. 2004. Recherches botaniques et écologiques sur les forêts actuelles du Bénin. Université de Cocody, Côte d'Ivoire, p.325.

Akoègninou A, van der Burg WJ, Maesen LJG. 2006. Flore Analytique du Bénin. Backhuys Publishers: Wageningen.

Akpo LE. 1993. Effet de l'Arbre sur la Structure et le Fonctionnement de la Strate Herbacée en Milieu Sahélien. Orstom éd., TDM 93 F2.

Akpo LE. 1998. Effet de l'arbre sur la végétation herbacée de quelques phytocénoses au Sénégal. Variation selon un gradient de pluviosité. Doctorat d'état ès Sc. nat., p. 132.

Auclaire A, Goff FG. 1971. Diversity relation of upland forest in western Great Lakes Area. Am. Nat., 105: 300-320.
Braun-Blanquet J. 1932. Plant Sociology. The Study of Plant Communities, Fuller GD, Conard MS (eds).

FAO. 2003. Situation des Forêts du Monde ( $5^{\text {ème }}$ Edition). FAO : Rome, Italie ; $151 \mathrm{p}$.

Frontier S, Pichod-Viale D. 1995. Ecosystèmes : Structure, Fonctionnement, Evolution $\left(2^{\mathrm{e}} \quad\right.$ Edition). Collection d'Ecologie 21, Masson : Paris.

Gillaumet JL. 1967. Recherches sur la végétation et la flore de la région du bascavally (Côte d'Ivoire). Mémoires ORSTOM N²0, Paris.

Hountondji YC. 1998. Contribution à l'étude des possibilités d'aménagement de la forêt naturelle de Niaouli au Sud du Bénin. Mémoire du DIT/CPU/UNB, p. 164.

Legendre, Legendre P. 1984. Ecologie Numérique: La Structure des Données Ecologiques (Tome 2). Masson Collection d'Écologie $\mathrm{n}^{\circ} 13$.

Raunkiaer C. 1934. The Life forms of Plants and Statistical Plant Geography. Clarendron Press: London.

Sokpon N. 1995. Recherche écologique sur la forêt dense semi-décidue de Pobè au Sudest du Bénin; groupements végétaux, structure, régénération naturelle et chute de litière. Thèse de doctorat, ULB, p. 350.

White F. 1986. La végétation de l'Afrique. Mémoire accompagnant la carte de végétation de l'Afrique. UNESCO/ AETFAT/UNSO ORSTOM-UNESCO. 